\title{
Epúlide canino: importância e aspectos clínico-histológicos
}

\section{Canine epulides: importance and clinical and histological aspects}

\author{
Fabiana Pizzolato de Lucena, ${ }^{\star}$ Renata Falcão Rabello da Costa, ${ }^{\star \star}$ Flávia Liparisi, ${ }^{\star \star \star}$ \\ Rogério Tortelly, ${ }^{\star \star *}$ Eulógio Carlos Queiroz de Carvalho***
}

\begin{abstract}
Resumo
Há muito, a expressão epúlide foi usada genericamente na clínica para qualquer crescimento gengival sendo, entretanto, uma neoplasia benigna do estroma ligamentar periodontal. Foram revistos, para estudo comparativo, 31 casos de neoplasias orais em cães, encaminhadas ao Setor de Anatomia Patológica Veterinária da UFF e ao Instituto de Medicina Veterinária Jorge Vaitsman /RJ, diagnosticados como epúlide odontogênico. Seguindo a classificação de Dubielzig et al., (1979), 16 casos foram diagnosticados como epúlide fibromatoso, oito casos como epúlide acantomatoso e sete casos como epúlide ossificante. A classificação histológica e o comportamento biológico, com vistas ao prognóstico de cada tipo desta neoplasia são revisados e discutidos.
\end{abstract}

Palavras-chave: epúlide, cavidade oral, canino.

\begin{abstract}
The word epulides has already been used as a clinical term for any gingival growing mass, although it is actually a benign neoplasm originated from the stroma of periodontal ligamentum. Thirty-one cases of oral neoplasms in dogs, sent to Setor de Anatomia Patológica Veterinária /UFF and to Instituto de Medicina Veterinária Jorge Vaitsman / RJ, diagnosed as odontogenic epulides, were reviewed for comparative study. Following the Dubielzig et al., (1979) classification, 16 cases were diagnosed as fibromatous epulides, 8 cases as acanthomatous epulides and 7 cases as ossifying epulides. The histological classification is of great value to evaluate the behavior and the prognosis of each kind of this neoplasm.
\end{abstract}

Keywords: epulide, oral cavity, canine.

\section{Introdução}

Diferentes lesões proliferativas acometem a cavidade oral de cães e gatos, devendo ser precisa e diferencialmente diagnosticadas, para o tratamento e prognóstico pertinentes (Dubielzig, 1982).

Designa-se hiperplasia gengival o crescimento do estroma que pode, assim como as lesões inflamatórias, simular neoplasias orais que não são comuns nestas espécies (Dubielzig, 1982). A cavidade oral é o quarto sítio de maior ocorrência de neoplasias em cães, afetando principalmente os machos (Oakes et al., 1993; Baker et al., 1993). O melanoma maligno, o fibrossarcoma e o carcinoma epidermóide são tumores comuns, representando $50 \%$ das neoplasias orais mais observadas (Dubielzig et al., 1979; Oakes et al., 1993). O epúlide destaca-se entre as neoplasias benignas, contribuindo com 25\% dos casos (Oakes et al., 1993).

O termo epúlide ou épulis é descrito clinicamente como um crescimento não ulcerado da margem de gengiva, comum nos cães e raro em outras espécies. Sua natureza exata deve ser determinada histologicamente (Harvey, 1992), já que se assemelha a neoplasias malignas como o carcinoma epidermóide e o fibrossarcoma. Acomete os dois sexos, havendo predisposição racial em boxer e bulldog (Harvey, 1992; Rodrigues Guirós et al., 1999) e sendo mais observado em animais entre sete e oito anos (Head, 1990).

Macroscopicamente, o epúlide se apresenta como pequenas massas, às vezes pedunculadas, circulares ou alongadas, com consistência de acordo com sua constituição. Na maioria dos casos não há sinais clínicos nem interferência na mastigação. Quando o crescimento ultrapassa o dente, atingindo a arcada oposta e prejudicando a mastigação, recomenda-se a extração cirúrgica.

O epúlide se origina do estroma do ligamento periodontal, ocorrendo na gengiva, principalmente próximo aos dentes molares (Dubielzig et al., 1979; Dubielzig, 1982; Jones et al.. 2000; Oakes et al., 1993). Atualmente, são classificados em três tipos: epúlide fibromatoso, epúlide ossificante e epúlide acantomatoso, que têm em comum, além da origem periodontal, estroma com colágeno denso e fibrilar, células estrelares dispostas a distância regular e intensa vascularização (Dubielzig et al., 1979; Dubielzig, 1982; Baker et al., 1993; Van Kruiningen, 1998).

\footnotetext{
* Médica-veterinária / Instituto de Medicina Veterinária Jorge Vaitsman / RJ.

** Mestranda do Programa de Pós-graduação em Medicina Veterinária / UFF.

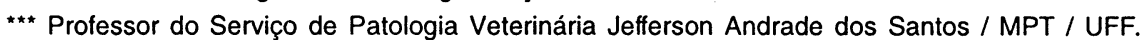


Os tipos fibromatoso e ossificante são bastante semelhantes sob o aspecto clínico e histológico, havendo no ossificante maior quantidade de matriz osteóide associado ao estroma ligamentar (Baker et al., 1993). Usualmente não são invasivos, e radiograficamente podem se apresentar mais ou menos mineralizados, de acordo com o tipo, com deslocamento dentário e, ocasionalmente, com osteólise (Oakes et al., 1993). Quando existe o envolvimento dentário e comprometimento da mastigação pela tumoração é indicada a crioterapia, a radioterapia ou a excisão cirúrgica completa, para prevenir a recidiva.

O tipo acantomatoso, apesar de ser considerado benigno, por não apresentar metástases, tem grande poder infiltrativo. São localmente agressivos, iniciam com lesão discreta, mas podem chegar a causar destruição de ossos adjacentes, o que pode ser notado ao exame radiográfico (Gardner, 1993). Além dos tratamentos citados, em alguns casos se recomenda a mandibulectomia ou maxilectomia parciais. A invasão óssea aumenta o risco de recidiva. A ocorrência de tumores malignos, principalmente o carcinoma epidermóide, no sítio do epúlide acantomatoso previamente irradiado, é uma complicação que os clínicos envolvidos nesta terapia devem estar conscientes (Thrall, 1981).

Queremos ressaltar a importância do diagnóstico preciso e específico dos tipos de epúlides para, a par do comportamento biológico de cada tipo, poder o clínico firmar prognóstico e tratamento adequados.

\section{Material e métodos}

Foram revistos 31 casos de neoplasias de cavidade oral de cães de diferentes idades e raças. O material avaliado foi obtido de levantamento de casos diagnosticados como epúlide odontogênico, no Instituto Municipal de Medicina Veterinária Jorge Vaitsman e na Faculdade de Veterinária da Universidade Federal Fluminense.

As amostras foram processadas por inclusão em parafina e coradas pela hematoxilina-eosina, sendo observadas em microscópio óptico e avaliadas de acordo com Dubielzig et al. (1979). Foram classificadas em três tipos: epúlide fibromatoso, epúlide ossificante e epúlide acantomatoso.

\section{Resultados}

Os 31 casos de epúlides odontogênicos corresponderam a: $51,6 \%$ de epúlide fibromatoso (16 casos), 25,8\% de epúlide acantomatoso (oito casos) e $22,6 \%$ de epúlide ossificante (sete casos).

$\mathrm{Na}$ maioria dos casos, as massas tumorais se localizaram, na gengiva, próximo aos dentes pré-molares e molares (Figura 1).

O tipo fibromatoso foi o mais freqüente. Caracterizou-se, microscopicamente, pela predominância de estroma denso, apresentando células de formato estrelado e colágeno fibrilar, lembrando o ligamento periodontal. A vascularização era intensa e distribuída regularmente (Figura 2). Foram encontrados restos de epitélio laminar dental, pequenos agregados de tecido osteóide, dentina ou cemento. Em alguns casos havia, ainda, discreta quantidade de tecido ósseo.

O epúlide acantomatoso foi representado, microscopicamente, por grandes extensões de tecido epitelial acantomatoso,

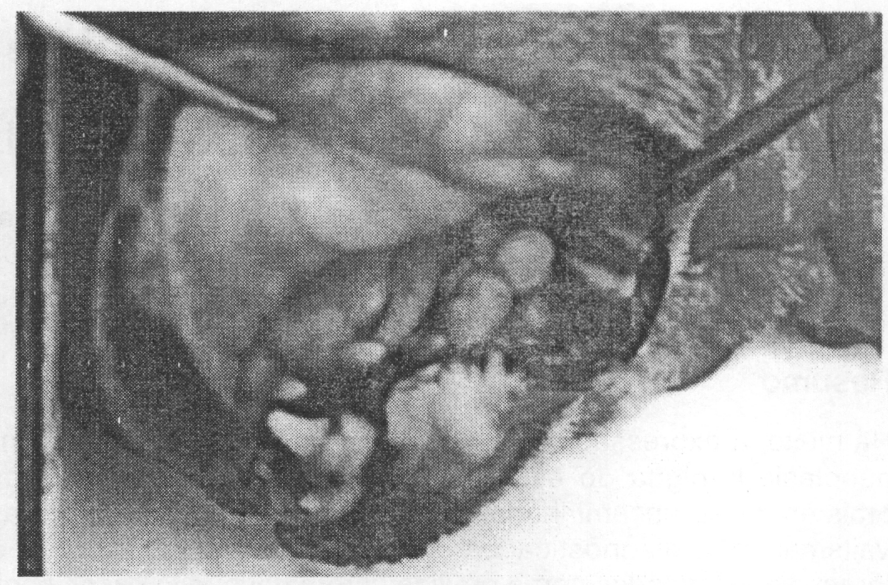

Figura 1: Canino - Epúlide - Massa gengival entre dentes pré-molares.

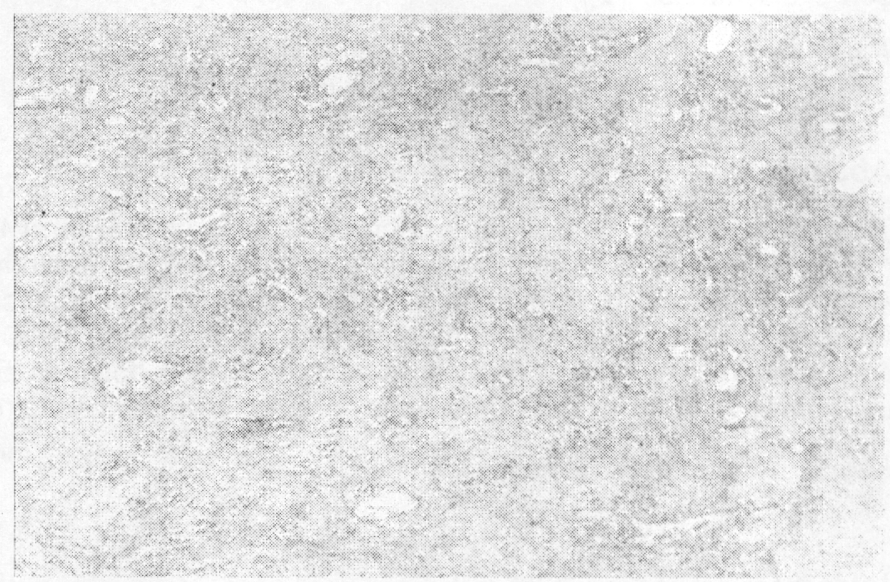

Figura 2: Epúlide fibromatoso - Rica vascularização no ligamento periodontal (HE 100X).

disposto em lâminas e cordões sólidos que se projetavam no estroma do ligamento periodontal. As células epiteliais ordenavam-se "em paliçada" ao longo da membrana basal (Figura 3). Pontes intercelulares eram comumente conspícuas podendo a invasão óssea ser observada.

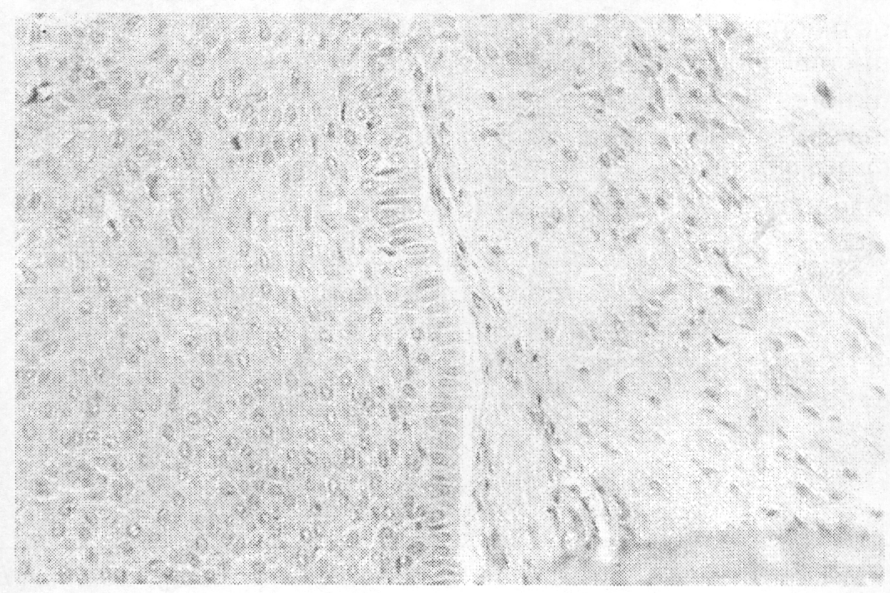

Figura 3: Epúlide acantomatoso - Células epiteliais dispostas "em paliçada" ao longo da membrana basal (HE 200X). 
O epúlide ossificante foi o menos observado, assemelhandose ao tipo fibromatoso nas características microscópicas, porém com maior quantidade, usualmente predominante, de matriz osteóide associada ao ligamento periodontal (Figura 4).

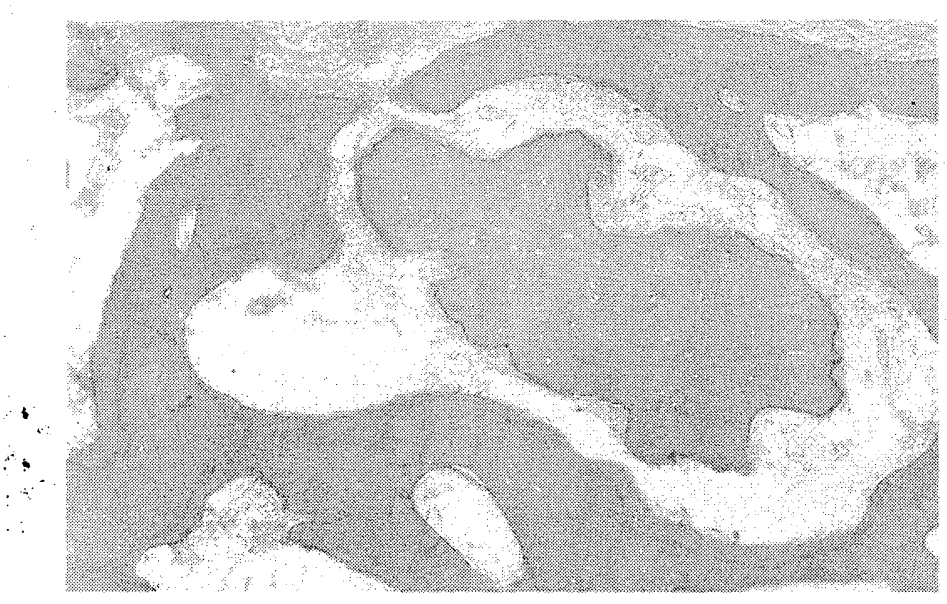

Figura 4: Epúlide ossificante - Ligamento periodontal associado à matriz osteóide (HE 200X).

\section{Discussão}

Uma classificação distinta da utilizada neste trabalho é sugerida por Vestraete et al. (1992), que utilizam as denominações hiperplasia focal fibrosa e fibroma odontogênico para os casos classificados como epúlide fibromatoso ou ossificante. Acreditam ainda, que a natureza da lesão não seja neoplásica, e sim uma gengivite crônica, sendo a proliferação de fibroblastos e a hiperplasia epitelial, provavelmente, originárias da gengiva e não do ligamento periodontal, como propomos aqui. Foi sugerido que todos os casos classificados como epúlide fibromatoso ou ossificante fossem enquadrados como fibromas odontogênicos "periféricos", e o epúlide acantomatoso como carcinoma de células basais, originado de células da camada germinativa da mucosa oral (Bostock e White, 1987). Porém, aceitam que há infiltração óssea pela neoplasia e recidiva.

Adotamos a classificação estabelecida por Dubielzig et al. (1979), levando em consideração as características morfológicas de cada tipo de epúlide (Tabela 1 ). Vale ressaltar que no tipo

\section{Referências}

BARKER, I.K.; VANDREUMEL, A.A.; PALMER, N. The Alimentary System. In: JUBB, K.V.F.; KENNEDY, P.C.; PALMER, N. Pathology of Domestic Animals. 4.ed. California: Academic Press, 1993, cap.1, 2 v., p.1-318. DUBIELZIG, R.R.; GOLDSCHMIDT, M.H.; BRODEY, R.S. The Nomenclature of Periodontal Epulides in Dogs. Veterinary Pathology, v. 16, n. 209, p. 214, 1979.

DUBIELZIG, R.R. Proliferative Dental and Gingival Diseases of Dogs and Cats. Journal of the American Animal Hospital Association, v. 18, p. 577-584, jul./ago. 1982.

GARDNER, D.G.; BAKER, D.C. The Relationship of the Canine Acanthomatous Epulis to Ameloblastoma. Journal of Comparative Pathology, v.108, p. 47-55, 1993.

HARVEY, C.E. Distúrbios orais, faringeanos e das glândulas salivares. In: ETTINGER, S.J. (Ed.) Tratado de Medicina Interna Veterinária. 3. ed. São Paulo: Manole, 1992. 1853 p. cap. 82, 3 v., p. 1265-1317. acantomatoso há uma característica bastante marcante, não incluída na tabela, que é a disposição das células epiteliais "em paliçada" ao longo da membrana basal.

Algumas vezes, este tipo pode ser erroneamente diagnosticado como ameloblastoma ou carcinoma epidermóide. A diferenciação dá-se, no primeiro caso, pela presença de mantos entrelaçantes de células epiteliais, com formação em paliçada na base desses mantos. No centro há formação semelhante a retículo estrelado, cordões entrelaçantes de células epiteliais, queratinização abrupta e intensa, freqüentemente com células grandes e redondas bastante queratinizadas. $\mathrm{O}$ ameloblastoma pode ser diferenciado do epúlide acantomatoso porque o estroma característico não é encontrado, não ocorre queratinização e há o achado específico de corpúsculos hialinos intercelulares, e no segundo, pela presença de atipia celular e pela ausência do ligamento periodontal (Van Kruiningen, 1998).

Em contraposição a Gardner e Baker (1993), consideramos o ameloblastoma uma neoplasia distinta de epúlide acantomatoso, basicamente por não apresentar o estroma do ligamento periodontal.

O resultado obtido no presente trabalho não reflete necessariamente a incidência do epúlide na população canina.

Neoplasias mais agressivas podem estar superestimadas por serem estas as que os clínicos veterinários mais se preocupam em encaminhar para exame histopatológico.

É muito importante para os clínicos que as tumorações retiradas da cavidade oral sejam enviadas para exame histopatológico, para permitir um prognóstico preciso.

Tabela 1: Características morfológicas das tumorações periodontais

\begin{tabular}{|c|c|c|c|}
\hline & $\begin{array}{l}\text { Epúlide } \\
\text { fibromatoso }\end{array}$ & $\begin{array}{c}\text { Epúlide } \\
\text { ossificante }\end{array}$ & $\begin{array}{c}\text { Epúlide } \\
\text { acantomatoso }\end{array}$ \\
\hline Estroma periodontal & + & + & + \\
\hline Osteóide & \pm & Abundante & \pm \\
\hline Cemento ou dentina & \pm & \pm & \pm \\
\hline Cordões do epitélio & \pm & \pm & \pm \\
\hline $\begin{array}{l}\text { odontogênico } \\
\text { Lâminas sólidas de células } \\
\text { epiteliais com proeminentes }\end{array}$ & & & \\
\hline pontes intercelulares & - & - & + \\
\hline Infiltração óssea local & - & - & \pm \\
\hline
\end{tabular}

+ =sempre presente; \pm = pode ou não estar presente; - = ausente Fonte: Dubielzig, Goldschmidt \& Brodey, 1979

HEAD, K.W. Tumors of the Alimentary Tract. In: MOULTON, J.E. (Ed.) Tumors in Domestic Animals. 3. ed. California: University of California Press, 1990.672 p. cap. 8, p. 347-435.

JONES, T.C.; HUNT, R.D.; KING, N.W. Sistema digestivo. Patologia Veterinária. 6. ed. São Paulo: Manole, 2000. 1415 p. cap. 23, p. 1063-1130. OAKES, M.G.; LEWIS, D.D.; HEDLUNG, C.S. et al. Canine Oral Neoplasia. The Compendium, v. 15, n. 1, p. 15-30, jan. 1993.

RODRIGUES GUIRÓS, J.; TROBO MUÑIZ, J.I.; COLLADO, J. et al. Neoplasia Orais em Pequenos Animais. In: SAN ROMÁN, F. (Ed.). Atlas de Odontologia de Pequenos Animais. São Paulo: Manole, 1999. cap. 9, 143-163.

THRALL, D.E.; GOLDSCHMIDT, M.H.; BIERY, D.V.M. Malignant Tumor Formation at the Site of Previously Irradiated Acanthomatous Epulides in Four Dogs. JAVMA, v. 178, n. 2, p. 127-132, jan. 1981.

VAN KRUININGEN, H.J. Sistema Gastrointestinal. In: CARTON, W.W.; Mc GAVIN, M.D. (Eds.) Patologia Veterinária Especial de Thomson. 2. ed. Porto Alegre: Artmed, 1998. 672 p. cap. 1, p. 13-94. 